\title{
"Mein wichtigstes Instrument ist mein eigener Körper»
}

\section{Daniel Lüthi}

Freier Journalist und Fotograf, Medientrainer, Bern

Wintersaison im Wallis. Physiotherapie ist da mit klaren Bildern verbunden: Hochbetrieb in einer blitzblanken Hightech-Praxis, Muskeltraining an modernen Maschinen, weissgewandete, sportliche Therapeutinnen geben verunglückten Ski- und Snowboard-Fahrern kurze Instruktionen, mit welchen Übungen sie welche Körperteile wieder in Schwung bringen können. Nichts von dem hier - diese Begegnung ist ein Kontrastprogramm.

\section{Affektive Abstimmung}

Das kleine Studio ist schwierig zu finden. Es liegt im Hinterhof eines herrschaftlichen Hauses in der Alt- stadt von Sion. Die beiden Zimmer erinnern eher an eine gemütliche Studentenloge als an ein klinisches Ambiente, sie sind umgeben von dicken, mittelalterlichen Mauern, die Decke wird getragen von dunklen Holzbalken. Das Wartezimmer ist mit einem starken, mediterranen Orange bemalt, das Behandlungszimmer mit Holzplatten isoliert. Eine Pflanze und ein eher düsteres Bild mit einer Eule wirken zufällig, es sind Patientengeschenke. Ein paar verwaiste Ordner und einige lose Papiere liegen herum, durch das Fenster fällt der Blick auf einen Feigenbaum und dahinter auf den Chor der nahen Kathedrale. In der Mitte des Raumes steht eine verstellbare Liege, in der Ecke eine Gitarre.

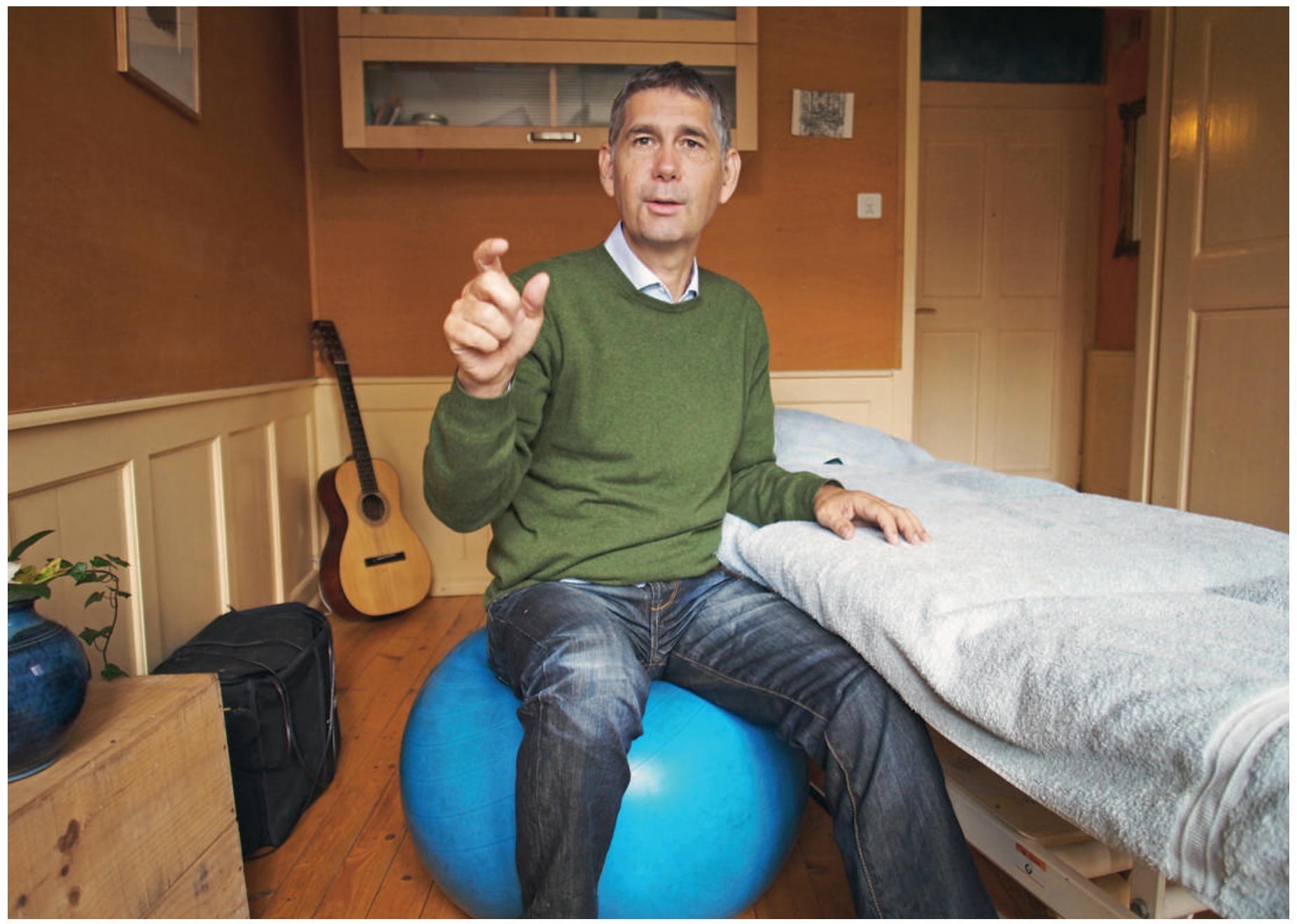


«Nein, kein Therapie-Instrument, sondern eine Entspannung für mich, ein Ausgleich in den Pausen», erklärt Urs Daendliker.

Eine Aussage mit durchaus symbolischem Charakter, wie sich bald zeigt. Denn auch, wenn er über seinen therapeutischen Ansatz spricht, denkt er ganz bewusst zuerst an seine eigene Befindlichkeit: «Ich kenne zwar das Alter, den Beruf und das Krankheitsbild eines Patienten, aber nur meine eigene Körpererfahrung orientiert mich darüber, was im Moment gerade geschieht. Wenn ich auf meinen eigenen Körper höre, erhalte ich wertvolle Informationen für den entscheidenden Moment der Kontaktaufnahme.» Der Psychoanalytiker Daniel Stern spreche in diesem Zusammenhang von einer "affektiven Abstimmung», erklärt Physiotherapeut Daendliker. «Mein Körper-Echo meldet mir Müdigkeit, Frustration oder Stress, bevor ich einem Patienten näherkomme, ihn berühre.» Er gebe also zuerst sich selber den nötigen Raum, bevor sich das Gefühl auf den Patienten übertragen könne, sich selber sein zu dürfen. «Eine Autorität ist einer, der autorisiert», philosophiert Urs Daendliker. "Unser Denken, unsere Analyse-Fähigkeit sind nur ein kleiner Teil eines globalen Systems. Mein wichtigstes Instrument ist mein eigener Körper.»

\section{Ein anderer Weg}

Kein Wort bisher also von schweisstreibender Gymnastik an genialen Geräten, von Triggerpunkt-, Elektro- oder Ultraschall-Behandlungen, kein Hinweis weiterhin auf Hanteln, Fitnessbänder oder Trampoline. Dafür die Einladung zu einer kleinen Entdeckungsreise durch den Körper. "Was fühlen Sie in Ihrem Knie?», würde er beispielsweise jemanden fragen, der nach einer Knieoperation zu ihm komme. «Fühlt es

\section{«Mein Körper-Echo meldet mir Müdigkeit, Frustration oder Stress.»}

sich an wie Stein - oder eher Holz? Metallisch vielleicht?» Es sei interessant, welche Bilder ein Patient produziere, und was allein dieser Prozess auslösen könne, sagt einer, der selber gerne in Bildern spricht. «Ich bin wie ein Bergführer, trage nicht den Rucksack des Patienten und gehe auch nicht an seiner Stelle.» Nein, mit Reden allein lasse sich kein Berg bezwingen und kein Knie heilen, antwortet Daendliker auf die entsprechende Frage, «aber der Weg ist nicht direkt. Wenn Sie Schmerzen haben, bauen Sie ein Schutzsystem auf, ein System geprägt von Angst und Vermeidung. Ich versuche, dem Patienten einen neuen Zugang zu seinem Körper zu öffnen.»

\section{Urs Daendliker}

Urs Daendliker wurde 1961 in Nyon geboren - als Kind eines Ehepaares aus Stäfa. In Nyon und Genf wuchs er auf. Nach der Matura absolvierte er die Physiotherapie-Schule in Genf, die er 1985 mit dem Diplom abschloss. Es folgten zahlreiche Weiterbildungen: So arbeitete er während eines Jahres am Genfer Kinderspital, er lernte genauer die Reflexzonenmassage kennen, die Sophrologie, die Mézières-Methode und die viszerale Osteopathie. 1990/91 arbeitete er für das IKRK mit Kriegsverletzten in Somalia und in Pakistan. 1992 eröffnete er seine eigene Praxis in Sion. Laufend bildet er sich weiter, so zum Beispiel in traditioneller chinesischer Medizin oder in der Feldenkrais-Methode. Er ist ein aktives Mitglied im nationalen Verband physioswiss, auch in der Qualitätskontrolle. Urs Daendliker ist verheiratet und Vater von zwei erwachsenen Kindern. Er lebt mit seiner Frau in Champlan, oberhalb von Sion.

Mit Verlaub: Das tönt alles ziemlich esoterisch. Ernsthafte Zweifel würden aufkommen, wäre es nicht der offizielle Berufsverband physioswiss gewesen, der gerade diesen Therapeuten empfohlen hat. Also weiter im Text. Wo beispielsweise sind die Hanteln geblieben? Oder die Maschinen, mit denen ein Knie wieder kräftig trainiert werden kann? «Kein Kind stemmt Hanteln», antwortet Daendliker in gleichbleibender Ruhe und Klarheit. «Bei Erwachsenen kann das gleich sein: Jemand, der sein Knie stärken will, geht vielleicht wieder die Treppe hoch, statt den Lift zu benützen. Entscheidend sind der Zugang, das Vertrauen.» Es folgt ein wohl entscheidender Satz: "Maschinen sind nicht grundsätzlich falsch. Sie sind bloss ein anderer Weg, als ich ihn mit meinen Patienten gehe.»

\section{Motion und Emotion}

Immer noch betone die moderne Physiotherapie die technischen Aspekte zu stark, ist Daendliker überzeugt: «Im Vordergrund stehen der Wille, das Messbare und die Kontrolle.» Weniger wichtig seien allzu oft die spontane Bewegung, die Fantasie und die Kreativität. «Organische Aspekte wie eine Entzündung oder biomedizinische Elemente wie Kraft und Funktion sind das eine. Für mich steht die Partizipation im Vordergrund. Die Frage zum Beispiel, wann und wie ein Patient wieder Fussball spielen kann.» Mechanische Übungen seien dabei weniger wesentlich als das Bewusstsein und die Möglichkeit, Bewegungen im Alltag wieder integrieren zu können. «Vieles, was uns auf diesem Weg unterstützt, haben wir nicht-medizinischen Fachleuten überlassen, dem Tanz- oder Tai-Chi-Lehrer beispielsweise. Obschon wir wissen, dass alles, was uns 
emotional bewegt, uns auch körperlich in Bewegung bringen kann. Emotion und Motion liegen sehr nahe beieinander.» Für alte Menschen mit Hüftproblemen könne es beispielsweise gut sein, die Tänze aus der Jugend wieder tanzen zu lassen, Foxtrott, Walzer oder Cha-Cha-Cha.

"Als Physiotherapeut habe ich ein wertvolles Privileg, das in unserer Kultur immer seltener geworden ist», sagt Daendliker, «ich darf Patienten berühren. Das ermöglicht einen nonverbalen Austausch - und dem Patienten, mit seinem eigenen Körper wieder in Kontakt zu kommen und ihm zu vertrauen.» Eine seiner Spezialitäten sei die sanfte Berührung und Bewegung der Bauchhaut, «eine Technik, die ich bei einem französischen Osteopathen gelernt habe und die grosse Auswirkungen auf den Atem hat, aber auch auf die Beweglichkeit des ganzen Rumpfs». Erfolgreich wende

\section{«Wir sollten einen direkten Zugang zur Bevölkerung haben.»}

er sie bei Kindern an, aber beispielsweise auch bei Tetraplegikern, bei Säuglingen oder Sterbenden. «Viele meiner Kolleginnen und Kollegen sind immer im gleichen Segment tätig», ergänzt er. «Sie können die Vielseitigkeit ihrer Fähigkeiten gar nie richtig entwickeln.» In seinem Berufsverband sei er schon ein Exot, gibt Daendliker schmunzelnd zu, «aber viele meiner Kollegen lassen sich von mir behandeln - und sie wissen, warum». Vielleicht, weil sie wissen, dass er beim Verband Tutoren für die Qualitätszirkel ausbildet. Vielleicht aber auch deshalb, weil er sich für jeden Patienten eine Stunde lang Zeit nimmt, und nicht gleichzeitig mehrere Kunden abfertigt.

\section{Physio- und Psychotherapie}

«Eine Patientin, die einen Unfall hatte, will zuerst einmal erzählen, wie dieser Unfall passiert ist», erzählt er. «So erfuhr ich letzthin von einer Feministin, dass sie nicht von irgendeinem Fahrzeug angefahren worden war, sondern von einem Militärjeep. In der Theorie ist ein solches Detail unwichtig. In der Praxis kann es entscheidend sein. Das Hauptproblem dieser Frau war nicht ihr Armbruch. Sondern eine posttraumatische Belastungsstörung.» Hoppla. Ein Physiotherapeut, der sich anmasst, auch Psychotherapeut zu sein? «Zuhören dürfen alle Therapeuten», ist die Antwort, «und eine Patientin als ganzen Menschen empfangen auch.» Wenn nötig, verweise er schon weiter, das sei klar, «ich sehe die rote Fahne».

Aber es sei falsch, dass jemand in der Regel nur via ärztliche Verordnung zu einem Physiotherapeuten kommen könne, nur dann also, wenn er oder sie objektiv krank sei. «Wir sollten einen direkten Zugang zur Bevölkerung haben.» Oft sei zum Beispiel eine kaputte Schulter nur ein Vorwand, und die Leute seien verspannt oder müde. «Wenn sich jemand nach drei Behandlungen wieder besser fühlt, ist das doch ok, oder?» So gesehen könnten Physiotherapeutinnen und -therapeuten in unserem Gesundheitssystem durchaus auch eine Gatekeeper-Funktion wahrnehmen, ist Daendliker überzeugt, «ja wir könnten sogar den Medizinern leichte Fälle abnehmen».

Wie erlebt dieser Mann Ärztinnen und Ärzte? «Meine Erfahrung ist, dass sie sehr beschäftigt und meistens unter Druck sind. Sie haben eine grosse Verantwortung - und stehen unter dem Druck, alles objektivieren und kategorisieren zu müssen. Wenn ich selber einen Arzt beanspruche, habe ich oft das Gefühl, dass ich eine zusätzliche Belastung bin für ihn.» Im Gegensatz zu einem Arzt müsse er die objektive und die subjektive Ebene nicht unterscheiden, «ich kann mit einem Patienten eine totale Allianz eingehen».

Beim Hinausgehen fällt der Blick auf das Logo dieser Praxis: die Skizze einer Figur in einem Balanceakt, den Kopf eher in den Wolken als gegen den Boden gerichtet. «Einer, der nicht in der Kontrolle der Situation ist, sondern in der Anwesenheit», kommentiert Urs Daendliker. «Einer, der besser geht, wenn er träumt. Ein Traumtänzer.» Das passt.

\section{Die nächste Begegnung mit ...}

Am Ende jeden Monats stellt die Schweizerische Ärztezeitung eine Persönlichkeit vor, die sich im Gesundheitswesen engagiert. Im März schildert Daniel Lüthi seine Begegnung mit Prof. Dr. Dr. Michael Thiel, Chefarzt der Augenklinik am Kantonsspital Luzern. 